\title{
The Metabolomic Paradigm of Pharmacogenomics in Complex Disorders
}

\section{Ramón Cacabelos ${ }^{1,2 *}$}

${ }^{1}$ EuroEspes Biomedical Research Center, Institute for CNS Disorders and Genomic Medicine, EuroEspes Chair of Biotechnology and Genomics, 15165-Bergondo, Corunna, Spain

${ }^{2}$ President, World Association of Genomic Medicine, Spain

Metabolomics represents the networking organization of multiple biochemical pathways leading to a physiological function in living organisms. The frontier between health and disease is likely to be the result of a fine-tuning equilibrium or disequilibrium, respectively, between the genomic-transcriptomic-proteomic-metabolomic cascade and environmental factors and/or epigenetic phenomena. In recent times, diverse metabolomic studies have emerged in medical science to explain physiological and pathogenic events in several disciplines, such as cardiovascular disorders [1], cancer [2], central nervous system (CNS) disorders [3,4] and drug development [5]; however, where metabolomics clearly depicts a prototypal paradigm of networking activity is in the confluence of multifactorial events responsible for the pharmacogenomic outcome [6]. Ideally, in the medical process, the onset of a particular disease should be followed by an understanding of its underlying pathogenic mechanism, an early diagnosis based on specific biomarkers, and a personalized treatment. Unfortunately, this is not the case in practice; with the exception of infectious diseases, about $80 \%$ of human diseases are of obscure etiopathogenesis (with poor understanding of their molecular mechanisms); their diagnoses lack specific biomarkers (especially in complex disorders), and their treatment is symptomatic rather than anti-pathogenic. Furthermore, only $20-30 \%$ of the medication available is cost-effective in major problems of health (cardiovascular, cancer, CNS), associated with over $70 \%$ of morbi-mortality cases in the developed world. Despite still abundant technical problems, it is expected that the "-omics" revolution may help to overcome the present limitations [7].

Most complex disorders share some common features: (i) they are polygenic disorders in which genetic, epigenetic and environmental factors are involved; (ii) some of them follow a golden rule: the higher the number of genes affected, the earlier the onset of the disease, with a faster progression, and a poorer therapeutic response to conventional drugs; and the smaller the number of genes disturbed, the later the onset, with a slower progression and a more favourable therapeutic response to current treatments; (iii) all of them are costly for society, deteriorating the quality of life of sufferers and increasing disability; and (iv) practically, the vast majority of complex disorders are susceptible to pharmacogenomic intervention.

The optimization of therapeutics requires the establishment of new postulates regarding (i) the costs of medicines, (ii) the assessment of protocols for multifactorial treatment in chronic disorders, (iii) the implementation of novel therapeutics addressing causative factors, and (iv) the setting-up of pharmacogenomic strategies for drug development [8-10]. Personalized therapeutics based on individual genomic profiles implies the characterization of 5 types of gene clusters and their corresponding metabolomic profiles: (i) genes associated with disease pathogenesis; (ii) genes associated with the mechanism of action of drugs; (iii) genes associated with drug metabolism (phase I and II reactions); (iv) genes associated with drug transporters; and (v) pleiotropic genes involved in multifaceted cascades and metabolic reactions [6].

Over 6,000 genes distributed across the human genome are potentially associated with human diseases. Different clusters of pathogenic genes usually converge in metabolomic networks leading to specific pathogenic cascades responsible for disease phenotypes. The genomics of the mechanism of action of drugs has so far been neglected by the scientific community, and consequently less than $5 \%$ of FDA-approved drugs, with a pharmacologically-defined mechanism of action, have been studied in order to evaluate whether mutations in the genes encoding receptors or enzymes may affect efficacy and safety issues. Pleiotropic genes, involved in multiple pathogenic events, are ever more extensively studied in different pathologies. $A B C$ genes, especially $A B C B 1$ (ATP-binding cassette, subfamily $\mathrm{B}$, member 1; P-glycoprotein-1, P-gp1; Multidrug Resistance 1, MDR1) (7q21.12), ABCC1 (9q31.1), ABCG2 (White1) (21q22.3), and other genes of this family, encode proteins which are essential for drug metabolism and transport. The multidrug efflux transporters P-gp, multidrugresistance associated protein 4 (MRP4) and breast cancer resistance protein (BCRP), located on endothelial cells lining brain vasculature, play important roles in limiting the movement of substances into and enhancing their efflux from the brain. Transporters also cooperate with Phase I/Phase II metabolism enzymes by eliminating drug metabolites. Their major features are their capacity to recognize drugs belonging to unrelated pharmacological classes, and their redundancy, by which a single molecule can act as a substrate for different transporters. This ensures an efficient neuroprotection against xenobiotic invasions. The pharmacological induction of $\mathrm{ABC}$ gene expression is a mechanism of drug interaction, which may affect substrates of the up-regulated transporter, and overexpression of MDR transporters confers resistance to anticancer agents and CNS drugs [11,12]. Also of importance for CNS pharmacogenomics are transporters, encoded by genes of the solute carrier superfamily (SLC) and solute carrier organic (SLCO) transporter family, responsible for the transport of multiple endogenous and exogenous compounds, including folate (SLC19A1), urea (SLC14A1, SLC14A2), monoamines (SLC29A4, SLC22A3), aminoacids (SLC1A5, SLC3A1, SLC7A3, SLC7A9, SLC38A1, SLC38A4, SLC38A5, SLC38A7, SLC43A2, SLC45A1), nucleotides (SLC29A2, SLC29A3), fatty acids (SLC27A1-6), neurotransmitters (SLC6A2 (noradrenaline transporter), SLC6A3 (dopamine transporter), SLC6A4 (serotonin transporter, SERT), SLC6A5, SLC6A6, SLC6A9, SLC6A11, SLC6A12, SLC6A14, SLC6A15, SLC6A16, SLC6A17, SLC6A18, SLC6A19), glutamate (SLC1A6, SLC1A7) and others [6].

*Corresponding author: Ramon Cacabelos, Professor \& Chairman, EuroEspes Biomedical Research Center, Institute for CNS Disorders and Genomic Medicine, EuroEspes Chair of Biotechnology and Genomics, 15165-Bergondo, Corunna, Spain. Tel: +34-981-780505; Fax: +34-981-780511; E-mail: rcacabelos@euroespes.com

Received September 13, 2012; Accepted September 14, 2012; Published September 17, 2012

Citation: Cacabelos R (2012) The Metabolomic Paradigm of Pharmacogenomics in Complex Disorders. Metabolomics 2:e119. doi:10.4172/2153-0769.1000e119

Copyright: (c) 2012 Cacabelos R. This is an open-access article distributed under the terms of the Creative Commons Attribution License, which permits unrestricted use, distribution, and reproduction in any medium, provided the original author and source are credited. 
Pharmacogenomics accounts for $30-90 \%$ variability in pharmacokinetics and pharmacodynamics.Most studies on pharmacogenetics/pharmacogenomics have been performed during the past 20 years (over 12,000 references) with genes encoding enzymes of Phase I reactions (i.e. oxidation, reduction, hydrolysis) and Phase II conjugation reactions (i.e. acetylation, glucuronidation, sulphation, methylation), especially genes of the cytochrome $\mathrm{P} 450$ superfamily (mixed-function monooxygenases). Most CYP enzymes display highly significant ethnic differences, indicating that the enzymatic capacity of these proteins varies depending upon the polymorphic variants present in their coding CYP genes. The practical consequence of this genetic variation is that the same drug can be differentially metabolized according to the genetic profile of each subject, and that knowing the pharmacogenomic profile of an individual, his/her pharmacodynamic response is potentially predictable. This is the cornerstone of pharmacogenetics. The CYP2D6, CYP2C19, CYP2C9 and CYP3A4/5 genes and their respective protein products are the most representative examples of this category.

CYP2D6 is a $4.38 \mathrm{~kb}$ gene with 9 exons mapped on 22q13.2. Four RNA transcripts of 1190-1684 bp are expressed in the brain, liver, spleen and reproductive system, where 4 major proteins of $48-55 \mathrm{kDa}(439-494$ aa) are identified. This protein is known to metabolize as many as $25 \%$ of commonly prescribed drugs and over $60 \%$ of current psychotropics. The gene is highly polymorphic in the population. There are 141 CYP2D6 allelic variants of which $-100 \mathrm{C}>\mathrm{T},-1023 \mathrm{C}>\mathrm{T},-1659 \mathrm{G}>\mathrm{A}$, -1707delT, -1846G $>$ A, -2549delA, -2613-2615delAGA, -2850C $>\mathrm{T}$, $-2988 \mathrm{G}>\mathrm{A}$, and $-3183 \mathrm{G}>\mathrm{A}$ represent the 10 most important variants. Different alleles result in the extensive, intermediate, poor, and ultrarapid metabolizer phenotypes, characterized by normal, intermediate, decreased and multiplied ability to metabolize the enzyme's substrates, respectively. According to the database of the World Guide for Drug Use and Pharmacogenomics [13], 982 drugs are CYP2D6-related: 371 drugs are substrates, over 300 drugs are inhibitors, and 18 drugs are CYP2D6 inducers. In European subjects, extensive metabolizers (EMs) account for $55.71 \%$ of the population, whereas $34.7 \%$ are intermediate metabolizers (IMs), $2.28 \%$ poor metabolizers (PMs), and $7.31 \%$ ultrarapid metabolizers (UMs) [6,8-10].

CYP2C9 is a gene $(50.71 \mathrm{~kb})$ with 9 exons mapped on 10q24. An RNA transcript of $1860 \mathrm{bp}$ is mainly expressed in hepatocytes where a protein of $55.63 \mathrm{kDa}$ (490 aa) can be identified. Over 600 drugs are CYP2C9-related, 311 acting as substrates (177 are major substrates, 134 are minor substrates), 375 as inhibitors (92 weak, 181 moderate, and 102 strong inhibitors), and 41 as inducers of the CYP2C9 enzyme [13]. There are 481 CYP2C9 SNPs. By phenotypes, in the control population, PMs represent 7.04\%, IMs 32.39\%, and EMs 60.56\% [6,8-10].

CYP2C19 is a gene $(90.21 \mathrm{~kb})$ with 9 exons mapped on 10q24.1q24.3. RNA transcripts of $1901 \mathrm{bp}, 2395 \mathrm{bp}$, and $1417 \mathrm{bp}$ are expressed in liver cells where a protein of $55.93 \mathrm{kDa}(490 \mathrm{aa})$ is identified. Nearly 500 drugs are CYP2C19-related, 281 acting as substrates (151 are major substrates, 130 are minor substrates), 263 as inhibitors (72 weak, 127 moderate, and 64 strong inhibitors), and 23 as inducers of the CYP2C19 enzyme [13]. About 541 SNPs have been detected in the CYP2C19 gene. The frequencies of the 3 major CYP2C19geno-phenotypes in the control population are CYP2C19- ${ }^{\star} 1 /{ }^{*} 1$-EMs $68.54 \%, C Y P 2 C 19-{ }^{*} 1 /{ }^{*} 2$ IMs $30.05 \%$ and CYP2C19- ${ }^{*} /{ }^{*} 2$-PMs $1.41 \%[6,8-10]$.

CYP3A4 is a gene $(27.2 \mathrm{~kb})$ with 13 exons mapped on $7 \mathrm{q} 21.1$. RNA transcripts of $2153 \mathrm{bp}, 651 \mathrm{bp}, 564 \mathrm{bp}, 2318 \mathrm{bp}$ and $2519 \mathrm{bp}$ are expressed in intestine, liver, prostate and other tissues where 4 protein variants of $57.34 \mathrm{kDa}(503 \mathrm{aa}), 17.29 \mathrm{kDa}$ (153 aa), $40.39 \mathrm{kDa}(353$ aa), and $47.99 \mathrm{kDa}(420 \mathrm{aa})$ are identified. The human $C Y P 3 A$ locus contains the three CYP3A genes (CYP3A4, CYP3A5 and CYP3A7), three pseudogenes as well as a novel CYP3A gene termed CYP3A43. The gene encodes a putative protein with, between $71.5 \%$ and $75.8 \%$ identity to the other CYP3A proteins. The predominant hepatic form is CYP3A4, but CYP3A5 contributes significantly to the total liver CYP3A activity. This enzyme metabolizes over 1900 drugs, 1033 acting as substrates (897 are major substrates, 136 are minor substrates), 696 as inhibitors (118 weak, 437 moderate, and 141 strong inhibitors), and 241 as inducers of the CYP3A4 enzyme [13]. About 347 SNPs have been identified in the CYP3A4 gene (CYP3A4* $1 A$ : Wild-type), 25 of which are of clinical relevance [13].

The construction of a genetic map integrating the most prevalent CYP2D6+CYP2C19+CYP2C9 polymorphic variants in a trigenic cluster, yields 82 different haplotype-like profiles. These 82 trigenic genotypes represent 36 different pharmacogenetic phenotypes. According to these trigenic clusters, only $26.51 \%$ of the patients show a pure $3 \mathrm{EM}$ phenotype, $15.29 \%$ are $2 \mathrm{EM} 1 \mathrm{IM}, 2.04 \%$ are pure $3 \mathrm{IM}, 0 \%$ are pure $3 \mathrm{PM}$, and $0 \%$ are $1 \mathrm{UM} 2 \mathrm{PM}$ (the worst possible phenotype). This implies that only one-quarter of the population normally processes the drugs which are metabolized via CYP2D6, CYP2C9 and CYP2C19 (approximately $60 \%$ of the drugs in current use) [6,14-15].

By knowing the pharmacogenomic profiles of patients with complex disorders, it might be possible to obtain some of the following benefits related to efficacy and safety issues: (i) to identify candidate patients with the ideal genomic profile to receive a particular drug (ii) to adapt the dose in over $90 \%$ of the cases according to the condition of EM, IM, PM or UM (diminishing the occurrence of direct side-effects in 30$50 \%$ of the cases) (iii) to reduce drug interactions by $30-50 \%$ (avoiding the administration of inhibitors or inducers, able to modify the normal enzymatic activity on a particular substrate) (iv) to enhance efficacy, and (v) to eliminate unnecessary costs ( $>30 \%$ of pharmaceutical costs) derived from the consequences of inappropriate drug selection and the overmedication administered to mitigate adverse drug reactions [6].

To make complex disorders a global health priority in the coming years, conceptual and procedural changes are needed on several grounds, such as (i) political, administrative, economic, legal, ethical, industrial, regulatory and educational issues; (ii) the implantation of novel biomarkers (genomics, transcriptomics, proteomics, metabolomics) as diagnostic and therapeutic aids; (iii) the introduction of innovative therapeutics; (iv) the implementation of pharmacogenomics in the clinical practice in order to optimize therapeutics; and $(\mathrm{v})$ the promotion of selective preventive plans for the population at risk.

\section{References}

1. Senn T, Hazen SL, Tang WH (2012) Translating metabolomics to cardiovascular biomarkers. Prog Cardiovasc Dis 55: 70-76.

2. Nishiumi S, Kobayashi T, Ikeda A, Yoshie T, Kibi M, et al. (2012) A novel serum metabolomics-based diagnostic approach for colorectal cancer. PLoS One 7: e40459.

3. He Y, Yu Z, Giegling I, Xie L, Hartmann AM, et al. (2012) Schizophrenia shows a unique metabolomics signature in plasma. Transl Psychiatry 2: e149.

4. Oresic M (2012) Obesity and psychotic disorders: uncovering common mechanisms through metabolomics. Dis Model Mech 5: 614-620.

5. Trupp M, Zhu H, Wikoff WR, Baillie RA, Zeng ZB, et al. (2012) Metabolomics reveals amino acids contribute to variation in response to simvastatin treatment. PLoS One 7: e38386.

6. Cacabelos R (2012) Pharmacogenomics of central nervous system (CNS) drugs. Drug Dev Res. Doi:10.1002/ddr.21039. 
Citation: Cacabelos R (2012) The Metabolomic Paradigm of Pharmacogenomics in Complex Disorders. Metabolomics 2:e119. doi:10.4172/2153$0769.1000 \mathrm{e} 119$

7. Eckhart AD, Beebe K, Milburn M (2012) Metabolomics as a key integrator for "omic" advancement of personalized medicine and future therapies. Clin Trans Sci 5: 285-288.

8. Cacabelos R, Fernández-Novoa L, Martínez-Bouza R, McKay A, Carril JC et al. (2010) Future Trends in the Pharmacogenomics of Brain Disorders and Dementia: Influence of APOE and CYP2D6 Variants. Pharmaceuticals 3: 30403100.

9. Cacabelos R, Martínez R, Fernández-Novoa L, Carril JC, Lombardi V, et al. (2012) Genomics of dementia: APOE- and CYP2D6-related pharmacogenetics. Int J Alzheimers Dis 2012: 518901.

10. Cacabelos R, Martínez-Bouza R, Carril JC, Fernández-Novoa L, Lombardi V et al. (2012) Genomics and pharmacogenomics of brain disorders. Curr Pharm Biotechnol 13: 674-725
11. marquez $b$, van bambeke $f$ (2011) abc multidrug transporters: target for modulation of drug pharmacokinetics and drug-drug interactions. Curr Drug Targets 12: 600-620.

12. Haufroid V (2011) Genetic polymorphisms of atp-binding cassette transporters abcb1 and abcc2 and their impact on drug disposition. Curr Drug Targets. 12: 631-646.

13. http://www.wgpgx.com/

14. Cacabelos R (2008) Pharmacogenomics in Alzheimer's disease. Methods Mo Biol 448: 213-357.

15. Cacabelos R (2009) Pharmacogenomics and therapeutic strategies for dementia. Expert Rev Mol Diagn 9: 567-611. 